\title{
Challenges in Radiotherapy
}

\author{
Stefanie Corradini ${ }^{a}$ David Krug ${ }^{b}$ Icro Meattinic, d Gerd Fastner $^{\mathrm{e}}$ \\ Christiane Matuschek ${ }^{f}$ Bruno Cutulig \\ ${ }^{\text {a } D e p a r t m e n t ~ o f ~ R a d i a t i o n ~ O n c o l o g y, ~ U n i v e r s i t y ~ H o s p i t a l, ~ L M U ~ M u n i c h, ~ M u n i c h, ~ G e r m a n y ; ~}{ }^{\text {b } K l i n i k ~ f u ̈ r ~}$ \\ Strahlentherapie, Universitätsklinikum Schleswig-Holstein, Campus Kiel, Kiel, Germany; ${ }^{\mathrm{C}}$ Department of \\ Biomedical, Experimental and Clinical Sciences, University of Florence, Florence, Italy; ${ }^{\mathrm{d}}$ Radiation Oncology Unit,

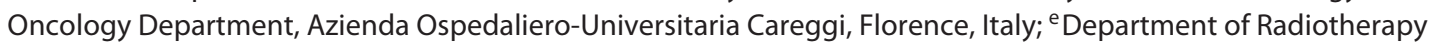 \\ and Radio-Oncology, Paracelsus Medical University, University Hospital Salzburg, Landeskrankenhaus, Salzburg, \\ Austria; fDepartment of Radiation Oncology, Heinrich Heine University Düsseldorf, Düsseldorf, Germany; \\ gPolyclinique Courlancy, Reims, France
}

\section{Question 1: What Treatment Do You Recommend after Complete Pathological Response after Neoadjuvant Chemotherapy in Initially Node Positive Breast Cancer (Biopsy Proven vs. Clinically Only)? Which Risk Factors Do You Take into Account for Your Decision in Favor of Postoperative Radiotherapy?}

Fastner: Up to now, there existed no clinical evidence to omit adjuvant locoregional radiotherapy (RT) of initial node positive $(\mathrm{cN}+)$ breast cancer patients, who develop a pathological complete response after neoadjuvant chemotherapy (NAC) followed by surgery $[1,2]$. This seems to be independent from whether $\mathrm{cN}+$ is histologically confirmed by biopsy or described according to radiological investigation only (ultrasound, MRT). However, if there remains uncertainty how to classify the clinical nodal status, either into $\mathrm{cN} 0$ or $\mathrm{cN}+$, a consecutive node biopsy is desirable [1]. In terms of breast-conserving surgery (BCS) and axillary dissection (level I/II), RT should be given to the whole breast and at least supra/infraclavicular nodes. Dependent on an individual case-by-case basis, regional node irradiation (RNI) should be expanded to axillary (insufficient axillary dissection, sentinel lymph node biopsy only) [3] or parasternal nodes (me$\mathrm{dial} /$ central tumor location and/or at least 3 clinical positive nodes) $[4,5]$, respectively. After mastectomy and axillary dissection, the indication for locoregional irradia- tion (chest wall and regional nodes) should be set in dependency of the initial clinical tumor stage and the presence of one or more risk factors [6, 7]. Hence, a premenopausal status, high-grade tumors (G3), a negativity for hormonal receptors or positivity for Her2neu, medial/ central tumor localization, lymphovascular invasion (L1) and $\mathrm{Ki}-67>30 \%$, either separately or in combination, should be taken into consideration for decision-making whether or not to irradiate in a specific case.

Krug: Unfortunately, there are no data from prospective randomized controlled trials to answer this question. There are two treatment decisions, which merit consideration: RNI and postmastectomy RT (PMRT). A recent systematic review showed that there are only few retrospective studies addressing the use of treatment response to NAC to guide decision-making regarding adjuvant RT in these two specific situations [1]. In general, PMRT and RNI were beneficial especially in patients with clinical stage III disease, even in the context of pathological complete response. However, data were conflicting in patients with stage II disease (i.e., T1-2 N1). I believe that PMRT and RNI should be standard in patients with clinical stage III disease and should be offered to all patients with clinical stage II disease in the presence of further risk factors, such as grade 3 , triple negative breast cancer, age $<40$ years. Suspicious lymph nodes should be histologically evaluated before initiation of treatment in all patients.

\section{KARGER}

(C) 2019 S. Karger AG, Basel
PD Dr. Stefanie Corradini

Department of Radiation Oncology, University Hospital, LMU Munich Marchioninistrasse 15

DE-81377 Munich (Germany)

E-Mail stefanie.corradini@med.uni-muenchen.de 
Matuschek: Adjuvant RT of the breast/chest wall and regional lymph node region $(\geq 3$ positive sentinel lymph nodes) after complete pathological response is recommended in the presence of high-risk factors including premenopausal hormonal status, central or medial tumor localization, high tumor grade (G2-3) and negativity for hormonal receptors.

Meattini: There is still no consensus on the use of postoperative RT in case of pathological complete response after NAC. In the largest reported analysis of RT for clinical N1 patients treated with NAC, PMRT was associated with improved overall survival for all pathological nodal subgroups, while no overall survival differences were observed with the addition of RNI to breast RT [8]. Several independent predictors of local relapse (LR) after BCS or mastectomy were identified, such as age, clinical nodal status (before NAC), and pathological nodal status/breast tumor response. By using these independent predictors, risk groups of patients could be identified and used to predict LR risk and to optimize the use of adjuvant RT [9].

In our institution, we do not routinely irradiate the chest wall or residual breast plus RNI after BCS or mastectomy in case of pathological complete response after NAC. However, we evaluate all patients who are candidates for NAC due to node positivity (both biopsy proven or clinically) for postoperative RT. For instance, we strongly recommend discussing each patient in a multidisciplinary tumor board, both pre- and postoperatively. Main characteristics to consider are initial burden of disease in the nodal regions and the breast (i.e., clinical stage III, cN2), age of patients (i.e., age less than 40 years), and tumor biology (i.e., triple negative, HER2-positive).

However, there is a lack of evidence from randomized trials, and major ongoing trials (i.e., NSABP B-51/RTOG 1304, Alliance 011202 trial) will answer many important questions, but additional issues will need to be addressed. Present consensus guidelines are based on retrospective studies or extrapolated data in the absence of level I evidence. Therefore, the ideal approach should be to enroll eligible patients in clinical trials.

\section{Question 2: Should the Internal Mammary Lymphatics Always Be Included in the Radiotherapy Volumes of Node Positive Breast Cancer Patients? Based on which Factors Do You Decide to Treat the Infra-/Supraclavicular Lymph Nodes Only and when Do You Include the Internal Mammary Nodes?}

Fastner: For patients with positive lymph nodes, RNI to the supra-/infraclavicular (SCV) and/or internal mammary region has shown to be beneficial with regard to survival end point analyses [9]. For a treatment decision, the following criteria should be evaluated: the number of affected lymph nodes ( $1-3$ vs. $\geq 4)$, tumor localization within the breast (central/inner or outer quadrants), tumor grade (intermediate to high or low grade), hormonal receptor status (positive or negative), and menopausal status (pre- or postmenopausal). The risk factor $\geq 4$ positive lymph nodes on its own, or 1-3 positive lymph nodes together with a central/inner tumor localization, RNI should be administered to both supraclavicular and internal mammary lymph nodes (IMN), respectively [10]. In all other cases dependent on tumor localization and at least two other risk factors (premenopausal status, negative hormonal receptors, intermediate or high tumor grading) RNI is considered for supraclavicular lymph nodes only.

Krug: Improvement of distant recurrence-free and disease-free survival has only been shown in trials which targeted the IMN along with the SCV lymphatic drainage [4, $10,11]$. Thus, I believe that the IMN should be treated, whenever improvement of these oncological end points is the goal. RNI may be used in patients with positive sentinel lymph node biopsy as an alternative to axillary dissection. The AMAROS trial did show similar axillary recurrence rates for RNI to the full axilla (level I-III) and the SCV lymph nodes as compared to axillary dissection in this setting [3]; however, lymph edema rates were cut in half with RNI.

Currently, there is no clear definition of prognostic or predictive factors that may guide the target definition in this situation. I would use comprehensive RNI including the IMN in patients with biologically aggressive tumors (triple negative breast cancer, high tumor grade), in patients with 4 or more involved lymph nodes and in patients with medial/central tumor location irrespective of the number of involved lymph nodes. In patients with positive sentinel lymph node biopsy without further risk factors, I would not treat the IMN. I do not routinely use RNI in node-negative patients. Whenever targeting the IMN, constraints for lung and heart should be carefully examined [12].

Matuschek: In the case of $\geq 3$ positive lymph nodes we indicate lymph node irradiation of the supra/infraclavicular lymphatic pathways and include the internal mammary lymphatic pathways in case of G2-3- or ER/PgRnegative tumors. In patients with 1-3 positive lymph nodes, we irradiate the SCV lymph node region when other high-risk factors are present: central or medial tumor localization and G2-3- or ER/PgR-negative or premenopausal patients with lateral localized breast cancer and G2-3 or ER/PgR-negative ones. The indication for RNI of the IMN pathway is the same in case of $1-3$ posi- 
tive lymph nodes, so we treat SCV and the IMN pathways as a package. We avoid irradiating the IMN pathway in case of systemic therapy with trastuzumab or in patients with high cardiac risk factors.

Meattini: In our institution we do not routinely irradiate IMN, except for cases where pre- or postoperative imaging shows IMN positivity or suspect lymph nodes. However, it has been recently demonstrated that the benefit of RNI including IMN for selected high-risk patients, with a marginal effect on overall survival but an improved disease-free, distant disease-free, and breast cancer-specific survival [4]. Major features to be considered should be: tumor biology, the dominant site of the breast lesion (i.e., medial localization), pathological T stage (i.e., pT2), and $\mathrm{N}$ stage (i.e., pN2). Therefore, the indication should always be discussed in a multidisciplinary team, considering also other postoperatively planned treatments.

\section{Question 3: Should Patients with Ductal Carcinoma in situ Always Receive Postoperative Radiotherapy following Breast-Conserving Surgery? What Is Your Clinical Practice in Your Country?}

Cutuli: In France, the national guidelines published in 2012 recommended postoperative RT after BCS for ductal carcinoma in situ (DCIS). In 2 large surveys, the rates of patients treated by RT after BCS were $89 \%$ (2003-2004) and 96\% (2014-2015) [13]. Indeed, 4 randomized trials and meta-analyses, as well as several other studies, showed a 50-60\% reduction of local recurrences (both in situ and invasive) with whole-breast irradiation (WBI; 50 Gy or equivalent dose).

Furthermore, even in extremely selected studies (ECOG-5194, RTOG-9804 [14]) for "very low-risk DCIS," the long LR rates are not negligible. Moreover, in case of invasive LR, approximately $12 \%$ of the patients developed long-term metastases. The RT omission should be debated in women older than 60 years with very small grade I DCIS, free margins ( $\geq 3 \mathrm{~mm}$ ), and favorable biological profile (e.g., positive HR, low Ki-67), such as proposed in a new "de-escalation" trial launched in France (ROMANCE Trial).

Fastner: According to the last meta-analysis of the early breast cancer trialists' collaborative group (EBCTCG) RT after BCS of DCIS halves the probability for breast recurrences in every risk group [15]. However, some controversy exists whether RT is mandatory in an exclusively low-risk constellation, like small tumor sizes $(<2.5 \mathrm{~cm})$, low-grade differentiation $(\mathrm{G} 1-2)$, and $>60$ years of age [16] which has been shown to be beneficial in terms of local control by the RTOG-9804 trial [14], respectively. Therefore, we are offering RT to all DCIS patients after BCS. Omission of RT should only be considered for frail elderly patients with a short expectancy of life.

Krug: I think that postoperative RT for patients with low-risk DCIS (tumor size $<2.5 \mathrm{~cm}, \mathrm{G1} / 2$, margins $>2$ $\mathrm{mm}$, age $>60$ years) should be discussed on a case-by-case basis. In the absence of a survival benefit and with a number needed to treat of around 12 for prevention of a DCIS recurrence and 25 for prevention of invasive recurrence [17], some of these women may opt to omit adjuvant RT. However, individual life expectancy, comorbidities, patient preferences and planned endocrine therapy as well as the excellent tolerability of adjuvant RT in the current era have to be considered [18]. In my experience, most women with DCIS have a strong wish for safety and choose to receive adjuvant RT. Nevertheless, shared decision-making and thorough communication of risks and benefits are crucial in this situation.

Matuschek: RT reduces the risk for a local recurrence significantly in DCIS but does not have any impact on overall survival. Especially in low-risk situations, we discuss the individual advantages and disadvantages of RT with every patient.

Meattini: RT reduces the absolute 10-year risk of any ipsilateral breast event (i.e., either recurrent DCIS or invasive cancer), and it is effective regardless of the age at diagnosis, extent of BCS, use of tamoxifen, method of DCIS detection, final surgical margin (FSM) status, focality, grade, comedonecrosis, architecture, or tumor size [15]. Moreover, this benefit persists over time [19, 20].

In our institution most patients still receive postoperative RT after BCS. It is well known that several patient-, tumor-, and treatment-related features significantly impact on LR risk [21]. Most patients with FSM less than $1 \mathrm{~mm}$ undergo re-excision. Therefore, the use of boost is not routinely performed since its ability to overcome the unfavorable effect of an unsatisfactory FSM is not clear [22]. The vast majority of patients did not receive an adjuvant endocrine therapy, since its benefit is less clear and robust as compared to adjuvant RT $[23,24]$.

However, a very-low-risk group of patients exists, for whom a de-escalation approach should be tested (i.e., radiation omission, partial breast RT). Since reliable and validated prognostic scores do still not exist $[25,26]$, a clinical trial context is preferable. 


\section{Question 4: Is the Hypofractionated Treatment Approach after Breast-Conserving Surgery Standard of Care in Your Country? Do You Apply Hypofractionation Also in the Treatment of Ductal Carcinoma in situ, after Mastectomy or in the Treatment of the Lymphatic Pathways?}

Cutuli: Hypofractionated whole-breast RT has been a validated option in France since 2015 (updated National Guidelines). According to international trials, three schemes are recommended, i.e. $42.5 \mathrm{~Gy} / 16$ fractions/3 weeks, $40 \mathrm{~Gy} / 15$ fractions/3 weeks, or $42 \mathrm{~Gy} / 13$ fractions/ 5 weeks. These RT modalities are preferably applied in postmenopausal patients with pT1T2N0 tumors. In these cases, the indications and modalities of a tumor bed boost remain widely debated. In the daily clinical practice for elderly patients ( $>70-75$ years), a protocol delivering a whole-breast dose of 45 Gy in 15 fractions over 5 weeks (3 fractions per week) is also commonly used. Another scheme developed in Nancy with a "concomitant boost" delivers $50 \mathrm{~Gy}$ in 25 fractions over 5 weeks, with twice a week an additional $1 \mathrm{~Gy}$ dose to the tumor bed (10 fractions in total, resulting in a whole radiobiological equivalent dose of $66 \mathrm{~Gy})$.

For DCIS, there are no official recommendations, but the results of several Canadian studies (Ontario and Quebec) confirmed the same local control between schemes of $50 \mathrm{~Gy} / 25$ fractions/5 weeks and $42.5 \mathrm{~Gy} / 16$ fractions/3 weeks. Regarding hypofractionation of the chest wall and/or lymphatic nodes, there are no specific recommendations due to scarce literature data.

Fastner: Hypofractionated WBI (HF-WBI) after BCS has proven to be at least isoeffective compared to conventional fractionation, in terms of local control, acute and late toxicity, for invasive breast cancer and DCIS, respectively $[27,28]$. Hence, HF-WBI is recommended as appropriate regimen primarily for patients who are not candidates for additional RNI. After mastectomy, a recently published prospective randomized trial reported no inferior clinical results if the chest wall and the ipsilateral supraclavicular/ level III axillary nodes were treated by moderate hypofractionation as compared to conventional standard irradiation [29]. This seems to be also true for hypofractionated $\mathrm{RNI}$ alone, if focusing on late reactions like plexopathia, shoulder immobility or arm lymphedema [30, 31]. According to the available data, some controversy exists for a common guideline-based treatment recommendation. However, on an individual case basis, moderate hypofractionated RNI seems possible [32]. On the basis of the current literature, in our institution HF-WBI is offered after BCS for invasive and in situ carcinoma of the breast as well as after mastectomy to the chest wall if indicated. Hypofractionated RNI is not performed outside of clinical trials.
Krug: The German national S3 guidelines recommend hypofractionated RT as the standard of care for postoperative whole-breast RT with the exception of patients receiving RNI [32]. The evidence regarding hypofractionated PMRT and RNI is still limited; however, data from a randomized controlled phase III trial have recently been published and support the use of hypofractionated RT in this setting [29]. I have used hypofractionation in selected patients with DCIS in the past; however, data from 2 randomized controlled phase III trials are still pending (TROG 07.01 (NCT00470236) and Bonbis (NCT00907868)).

Matuschek: Yes, the hypofractionated treatment in invasive breast cancer after BCS is standard of care in most German RT departments. When lymph node regions are included, maybe most departments still use the normofractionated regime. In the S3 guidelines the hypofractionated regime is recommended with more precaution because the hypofractionated irradiated patient group was underrepresented in the randomized trials. In DCIS after BCS we discuss the topic on an individual patient basis.

Meattini: Robust long-term data have demonstrated the noninferiority of HF-WBI as compared to conventional fractionated WBI $[33,34]$. Major updated guidelines stated that for women with invasive breast cancer receiving WBI, the preferred dose fractionation scheme is HF-WBI to a dose of $40 \mathrm{~Gy}$ in 15 fractions or $42.5 \mathrm{~Gy}$ in 16 fractions $[35,36]$.

HF-WBI is largely diffuse in Italy, although it is still not standard of care. In our institution we apply HF-WBI for all patients undergoing BCS for whom postoperative WBI is indicated, including DCIS patients (40 Gy in 15 fractions) since 2014 . When a tumor bed boost is prescribed, it is also hypofractionated (13.3 Gy in 5 fractions or 18.9 Gy in 7 fractions depending on FSM status). Conversely, after mastectomy or in the case of RNI we still use conventional fractionation (50 Gy in 25 fractions), waiting for the results from ongoing studies (i.e., Skagen trial 1, NCT02384733).

\section{Question 5: Do You Perform Partial Breast Irradiation? Which Patients Would You Treat with Partial Breast Irradiation and which Technique and Fractionation Do You Use?}

Fastner: Partial breast irradiation (PBI) either with postoperative hypofractionated external beam RT [37] intraoperative $[38,39]$ or interstitial techniques $[40,41]$ seems to be an alternative treatment option if patients could be allocated to "very" low-risk breast cancer subtypes [42-44]. In our own institution, intraoperative RT with electrons has had a long tradition administered as 
anticipated tumor bed boost since 1998. However, according to international guidelines and recommendations $[43,44]$, intraoperative RT with electrons as singleshot full-dose treatment (21 Gy at the $90 \%$ reference isodose, 23.4 Gy Dmax) is offered exclusively to patients who are classified as "suitable" with unicentric(-focal) Luminal A tumors of sizes $<2 \mathrm{~cm}$, negative lymph nodes (cN0 and negative intraoperative frozen section histology for sentinel nodes), $\geq 50$ years of age, clear resection margins, tumor grading G1/2 without neoadjuvant systemic treatment, respectively. Moreover, with lobular histology, preoperative breast MRT is mandatory [45]. Patients with pure DCIS or suspicious for an extensive/or predominant intraductal component and/or genetic high-risk constellation are not considered for PBI.

Krug: I do not routinely use accelerated PBI (APBI) in clinical practice. Nevertheless, I think that APBI may be offered to selected low-risk patients capable of understanding the limitations of the data currently available from randomized controlled trials. From my point of view, the preferred technique would be multicatheter brachytherapy with $8 \times 4$ Gy based on the excellent results from the GEC-ESTRO trial $[41,46]$ and the 10-year data from Hungary [47]. If multicatheter brachytherapy is not available, or institutional experience with this technique is limited, I would suggest percutaneous RT with $5 \times 6 \mathrm{~Gy}$ based on the Florence IMRT trial [48] or with $15 \times 2.67$ as per the IMPORT LOW trial [37].

Matuschek: Yes, but only in selected cases, especially older patients with low-risk breast cancer. In our breast cancer center intraoperative RT is possible. We discuss the advantages and disadvantages of PBI versus WBI or to omit RT with the patients. We offer $1 \times 20 \mathrm{~Gy}$ as a single fraction using intraoperative RT. For this procedure lead shielding for protection of the ribs is necessary.

Meattini: Following the published results of main phase III trials [37, 38, 41, 48, 49] we recommend PBI using external beam RT and brachytherapy techniques, in accordance with the UK consensus statement on postoperative RT for breast cancer [36]. Conversely, intraoperative RT is not recommended due to lack in robustness of published data (i.e., basic statistical assumptions, followup at time of publication, patient selection bias) [38, 4951].

In our Institution we follow the $30 \mathrm{~Gy}$ in 5 fractions schedule (6 Gy per fraction, every other day) using an intensity-modulated technique as described in the University of Florence phase III trial [48]. We strongly recommend to select patients by following the PBI ESTRO [40] and ASTRO [44] statements.

\section{Participants \\ Dr. David Krug \\ Klinik für Strahlentherapie \\ Universitätsklinikum Schleswig-Holstein, Campus Kiel \\ Arnold-Heller-Straße, 24105 Kiel, Germany \\ david.krug@uksh.de}

\section{Prof. Dr. Icro Meattini}

Department of Biomedical, Experimental and Clinical Sciences University of Florence

Viale Morgagni 85, 50134 Florence, Italy

icro.meattini@unifi.it

Dr. Gerd Fastner

Department of Radiotherapy and Radio-Oncology

Paracelsus Medical University, University Hospital Salzburg,

Landeskrankenhaus

Müllner Hauptstraße 48, 5020 Salzburg, Austria

g.fastner@salk.at

PD Dr. Christiane Matuschek

Department of Radiation Oncology

Heinrich Heine University Düsseldorf

Moorenstraße 5, 40225 Düsseldorf, Germany

Matuschek@med.uni-duesseldorf.de

Prof. Dr. Bruno Cutuli

Polyclinique Courlancy

38bis, rue de Courlancy, 51100 Reims, France

bcutuli@iccreims.fr

\section{References}

1 Krug D, Baumann R, Budach W, Dunst J, Feyer P, Fietkau R, et al. Individualization of post-mastectomy radiotherapy and regional nodal irradiation based on treatment response after neoadjuvant chemotherapy for breast cancer: a systematic review. Strahlenther Onkol. 2018 Jul;194(7):607-18. German.

2 Pazos M, Schönecker S, Reitz D, Rogowski P, Niyazi M, Alongi F, et al. Recent Develop- ments in Radiation Oncology: An Overview of Individualised Treatment Strategies in Breast Cancer. Breast Care (Basel). 2018 Aug; 13(4):285-91.

3 Donker M, van Tienhoven G, Straver ME, Meijnen P, van de Velde CJ, Mansel RE, et al. Radiotherapy or surgery of the axilla after a positive sentinel node in breast cancer (EORTC 10981-22023 AMAROS): a ran- domised, multicentre, open-label, phase 3 non-inferiority trial. Lancet Oncol. 2014 Nov; 15(12):1303-10.

4 Poortmans PM, Collette S, Kirkove C, Van Limbergen E, Budach V, Struikmans H, et al.; EORTC Radiation Oncology and Breast Cancer Groups. Internal Mammary and Medial Supraclavicular Irradiation in Breast Cancer. N Engl J Med. 2015 Jul;373(4):317-27. 
5 Hennequin C, Bossard N, Servagi-Vernat S, Maingon P, Dubois JB, Datchary J, et al. Tenyear survival results of a randomized trial of irradiation of internal mammary nodes after mastectomy. Int J Radiat Oncol Biol Phys. 2013 Aug;86(5):860-6.

6 Bellon JR, Wong JS, Burstein HJ. Should response to preoperative chemotherapy affect radiotherapy recommendations after mastectomy for stage II breast cancer? J Clin Oncol. 2012 Nov;30(32):3916-20.

7 Corradini S, Bauerfeind I, Belka C, Braun M, Combs SE, Eckel R, et al. Trends in use and outcome of postoperative radiotherapy following mastectomy: A population-based study. Radiother Oncol. 2017 Jan;122(1):210.

8 Rusthoven CG, Rabinovitch RA, Jones BL, Koshy M, Amini A, Yeh N, et al. Predictors of locoregional recurrence after neoadjuvant chemotherapy: results from combined analysis of National Surgical Adjuvant Breast and Bowel Project B-18 and B-27. J Clin Oncol. 2016;27:3960-6.

9 Mamounas EP, Anderson SJ, Dignam JJ, Bear HD, Julian TB, Geyer CE Jr, et al. Predictors of locoregional recurrence after neoadjuvant chemotherapy: results from combined analysis of National Surgical Adjuvant Breast and Bowel Project B-18 and B-27. J Clin Oncol. 2012 Nov;30(32):3960-6. Available from ht t p://ascopubs.org/doi/10.1200/ JCO.2011.40.8369

10 Thorsen LB, Offersen BV, Danø H, Berg M, Jensen I, Pedersen AN, et al. DBCG-IMN: A Population-Based Cohort Study on the Effect of Internal Mammary Node Irradiation in Early Node-Positive Breast Cancer. J Clin Oncol. 2016 Feb;34(4):314-20.

11 Whelan TJ, Olivotto IA, Parulekar WR, Ackerman I, Chua BH, Nabid A, et al.; MA.20 Study Investigators. Regional Nodal Irradiation in Early-Stage Breast Cancer. N Engl J Med. 2015 Jul;373(4):307-16

12 Corradini S, Ballhausen $\mathrm{H}$, Weingandt $\mathrm{H}$, Freislederer P, Schönecker S, Niyazi M, et al. Left-sided breast cancer and risks of secondary lung cancer and ischemic heart disease: effects of modern radiotherapy techniques. Strahlenther Onkol. 2018 Mar;194(3):196205.

13 Cutuli B, Lemanski C, Fourquet A, de Lafon$\tan$ B, Giard S, Meunier A, et al. Breast-conserving surgery with or without radiotherapy vs mastectomy for ductal carcinoma in situ: french Survey experience. Br J Cancer. 2009 Apr;100(7):1048-54.

14 McCormick B, Winter K, Hudis C, Kuerer HM, Rakovitch E, Smith BL, et al. RTOG 9804: a prospective randomized trial for good-risk ductal carcinoma in situ comparing radiotherapy with observation. J Clin Oncol. 2015 Mar;33(7):709-15.

15 Davidson N, Gelber R, Piccart M, Pruneri G, Pritchard K, Ravdin P, et al. Overview of the randomized trials of radiotherapy in ductal carcinoma in situ of the breast. J Natl Cancer Inst Monogr. 2010;41:162-77.

16 Sagara Y, Freedman RA, Vaz-Luis I, Mallory MA, Wong SM, Aydogan F, et al. Patient Prognostic Score and Associations With Survival Improvement Offered by Radiotherapy
After Breast-Conserving Surgery for Ductal Carcinoma In Situ: A Population-Based Longitudinal Cohort Study. J Clin Oncol. 2016 Apr;34(11):1190-6.

17 McCormick B. Randomized trial evaluating radiation following surgical excision for “good risk" DCIS: 12-year report from NRG/ RTOG 9804. Int J Radiat Oncol Biol Phys. 2018;102(5):1603.

18 Corradini S, Pazos M, Schönecker S, Reitz D, Niyazi M, Ganswindt U, et al. Role of postoperative radiotherapy in reducing ipsilateral recurrence in DCIS: an observational study of 1048 cases. Radiat Oncol. 2018 Feb;13(1):25.

19 Wapnir IL, Dignam JJ, Fisher B, Mamounas EP, Anderson SJ, Julian TB, et al. Long-term outcomes of invasive ipsilateral breast tumor recurrences after lumpectomy in NSABP B-17 and B-24 randomized clinical trials for DCIS. J Natl Cancer Inst. 2011 Mar;103(6): 478-88.

20 Bijker N, Meijnen P, Peterse JL, Bogaerts J, Van Hoorebeeck I, Julien JP, et al. Breast-conserving treatment with or without radiotherapy in ductal carcinoma-in-situ: ten-year results of European Organisation for Research and Treatment of Cancer randomized phase III trial 10853 - a study by the EORTC Breast Cancer Cooperative Group. J Clin Oncol. 2006 Jul;24(21):3381-7.

21 Meattini I, Pasinetti N, Meduri B, De Rose F, De Santis MC, Franco P, et al. A national multicenter study on 1,072 DCIS patients treated with breast-conserving surgery and whole breast radiotherapy (COBCG-01 study). Radiother Oncol. 2019 Feb;131:208-14.

22 Meattini I, Livi L, Franceschini D, Saieva C, Meacci F, Marrazzo L, et al. Role of radiotherapy boost in women with ductal carcinoma in situ: a single-center experience in a series of 389 patients. Eur J Surg Oncol. 2013 Jun; 39(6):613-8.

23 Cuzick J, Sestak I, Pinder SE, Ellis IO, Forsyth $S$, Bundred NJ, et al. Effect of tamoxifen and radiotherapy in women with locally excised ductal carcinoma in situ: long-term results from the UK/ANZ DCIS trial. Lancet Oncol. 2011;12:21-9.

24 Allred DC, Anderson SJ, Paik S, Wickerham DL, Nagtegaal ID, Swain SM, et al. Adjuvant tamoxifen reduces subsequent breast cancer in women with estrogen receptor-positive ductal carcinoma in situ: a study based on NSABP protocol B-24 [Internet]. J Clin Oncol. 2012 Apr;30(12):1268-73. Available from: http://ascopubs.org/doi/10.1200/ JCO.2010.34.0141

25 MacAusland SG, Hepel JT, Chong FK, Galper SL, Gass JS, Ruthazer R, et al. An attempt to independently verify the utility of the Van Nuys Prognostic Index for ductal carcinoma in situ [Internet]. Cancer. 2007 Dec;110(12): 2648-53. Available from: http://doi.wiley. com/10.1002/cncr.23089

26 Di Saverio S, Catena F, Santini D, Ansaloni L, Fogacci T, Mignani S, et al. 259 Patients with DCIS of the breast applying USC/Van Nuys prognostic index: a retrospective review with long term follow up [Internet]. Breast Cancer Res Treat. 2008 Jun;109(3):405-16. Available from: http://link.springer.com/10.1007/ s10549-007-9668-7
27 Valle LF, Agarwal S, Bickel KE, Herchek HA, Nalepinski DC, Kapadia NS. Hypofractionated whole breast radiotherapy in breast conservation for early-stage breast cancer: a systematic review and meta-analysis of randomized trials. Breast Cancer Res Treat. 2017 Apr; 162(3):409-17

28 Montero A, Sanz X, Hernanz R, Cabrera D, Arenas M, Bayo E, et al. Accelerated hypofractionated breast radiotherapy: FAQs (frequently asked questions) and facts. Breast. 2014 Aug;23(4):299-309.

29 Wang SL, Fang H, Song YW, Wang WH, Hu C, Liu YP, et al. Hypofractionated versus conventional fractionated postmastectomy radiotherapy for patients with high-risk breast cancer: a randomised, non-inferiority, open-label, phase 3 trial. Lancet Oncol. 2019 Mar;20(3):352-60.

30 Guenzi M, Blandino G, Vidili MG, Aloi D, Configliacco E, Verzanini E, et al. Hypofractionated irradiation of infra-supraclavicular lymph nodes after axillary dissection in patients with breast cancer post-conservative surgery: impact on late toxicity. Radiat Oncol. 2015 Aug;10(1):177.

31 Haviland JS, Mannino M, Griffin C, Porta N, Sydenham M, Bliss JM, et al.; START Trialists' Group. Late normal tissue effects in the arm and shoulder following lymphatic radiotherapy: Results from the UK START (Standardisation of Breast Radiotherapy) trials. Radiother Oncol. 2018 Jan;126(1):155-62.

32 Wöckel A, Festl J, Stüber T, Brust K, Krockenberger M, Heuschmann PU, et al. Interdisciplinary Screening, Diagnosis, Therapy and Follow-up of Breast Cancer. Guideline of the DGGG and the DKG (S3-Level, AWMF Registry Number 032/045OL, December 2017) Part 2 with Recommendations for the Therapy of Primary, Recurrent and Advanced Breast Cancer. Geburtshilfe Frauenheilkd. 2018 Nov; 78(11):1056-88.

33 Haviland JS, Owen JR, Dewar JA, Agrawal RK, Barrett J, Barrett-Lee PJ, et al.; START Trialists' Group. The UK Standardisation of Breast Radiotherapy (START) trials of radiotherapy hypofractionation for treatment of early breast cancer: 10-year follow-up results of two randomised controlled trials [Internet]. Lancet Oncol. 2013 Oct;14(11):1086-94. Available from: https://linkinghub.elsevier.com/retrieve/pii/S1470204513703863

34 Whelan TJ, Pignol JP, Levine MN, Julian JA, MacKenzie R, Parpia S, et al. Long-term results of hypofractionated radiation therapy for breast cancer [Internet]. N Engl J Med. 2010 Feb;362(6):513-20. Available from: http:// www.nejm.org/doi/abs/10.1056/NEJMoa0906260

35 Smith BD, Bellon JR, Blitzblau R, Freedman G, Haffty B, Hahn C, et al. Radiation therapy for the whole breast: executive summary of an American Society for Radiation Oncology (ASTRO) evidence-based guideline. Pract Radiat Oncol. 2018;8:145-52.

36 Bloomfield B, Brunt M, Chan C, Coles C, Crellin $\mathrm{C}$, Dodwell D, et al. Postoperative radiotherapy for breast cancer: UK consensus statements [Internet]. R Coll Radiol Lond. 2016. Available from: https://www.rcr.ac.uk/system/files/publication/field publication files/ bfco2016_breast-consensus-guidelines.pdf 
37 Coles CE, Griffin CL, Kirby AM, Titley J, Agrawal RK, Alhasso A, et al.; IMPORT Trialists. Partial-breast radiotherapy after breast conservation surgery for patients with early breast cancer (UK IMPORT LOW trial): 5-year results from a multicentre, randomised, controlled, phase 3 , non-inferiority trial. Lancet. 2017 Sep;390(10099):1048-60.

38 Veronesi U, Orecchia R, Maisonneuve P, Viale G, Rotmensz N, Sangalli C, et al. Intraoperative radiotherapy versus external radiotherapy for early breast cancer (ELIOT): a randomised controlled equivalence trial. Lancet Oncol. 2013 Dec;14(13):1269-77.

39 Vaidya JS, Wenz F, Bulsara M, Tobias JS, Joseph DJ, Keshtgar M, et al.; TARGIT trialists' group. Risk-adapted targeted intraoperative radiotherapy versus whole-breast radiotherapy for breast cancer: 5-year results for local control and overall survival from the TARGIT-A randomised trial. Lancet. 2014 Feb; 383(9917):603-13.

40 Polgár C, Van Limbergen E, Pötter R, Kovács G, Polo A, Lyczek J, et al.; GEC-ESTRO breast cancer working group. Patient selection for accelerated partial-breast irradiation (APBI) after breast-conserving surgery: recommendations of the Groupe Européen de Curiethérapie-European Society for Therapeutic Radiology and Oncology (GEC-ESTRO) breast cancer working group based on clinical evidence (2009). Radiother Oncol. 2010 Mar; 94(3):264-73.

41 Strnad V, Ott OJ, Hildebrandt G, KauerDorner D, Knauerhase H, Major T, et al.; Groupe Européen de Curiethérapie of European Society for Radiotherapy and Oncology (GEC-ESTRO). 5-year results of accelerated partial breast irradiation using sole interstitial multicatheter brachytherapy versus wholebreast irradiation with boost after breast-conserving surgery for low-risk invasive and insitu carcinoma of the female breast: a randomised, phase 3, non-inferiority trial. Lancet. 2016 Jan;387(10015):229-38

42 Sedlmayer F, Sautter-Bihl ML, Budach W, Dunst J, Fastner G, Feyer P, et al.; Breast Cancer Expert Panel of the German Society of Radiation Oncology (DEGRO). DEGRO practical guidelines: radiotherapy of breast cancer I: radiotherapy following breast conserving therapy for invasive breast cancer. Strahlenther Onkol. 2013 Oct;189(10):825-33.

43 Leonardi MC, Maisonneuve P, Mastropasqua MG, Morra A, Lazzari R, Rotmensz N, et al. How do the ASTRO consensus statement guidelines for the application of accelerated partial breast irradiation fit intraoperative radiotherapy? A retrospective analysis of patients treated at the European Institute of Oncology. Int J Radiat Oncol Biol Phys. 2012 Jul; 83(3):806-13.

44 Correa C, Harris EE, Leonardi MC, Smith BD, Taghian AG, Thompson AM, et al. Accelerated Partial Breast Irradiation: Executive summary for the update of an ASTRO Evidence-Based Consensus Statement. Pract Radiat Oncol. 2017 Mar - Apr;7(2):73-9.

45 Silverstein MJ, Fastner G, Maluta S, Reitsamer $\mathrm{R}$, Goer DA, Vicini F, et al. Intraoperative radiation therapy: a critical analysis of the ELIOT and TARGIT trials. Part 1-ELIOT. Ann Surg Oncol. 2014 Nov;21(12):3787-92.

46 Polgár C, Ott OJ, Hildebrandt G, KauerDorner D, Knauerhase H, Major T, et al.; Groupe Européen de Curiethérapie of Euro- pean Society for Radiotherapy and Oncology (GEC-ESTRO). Late side-effects and cosmetic results of accelerated partial breast irradiation with interstitial brachytherapy versus whole-breast irradiation after breast-conserving surgery for low-risk invasive and in-situ carcinoma of the female breast: 5-year results of a randomised, controlled, phase 3 trial. Lancet Oncol. 2017 Feb;18(2):259-68.

47 Polgár C, Fodor J, Major T, Sulyok Z, Kásler M. Breast-conserving therapy with partial or whole breast irradiation: ten-year results of the Budapest randomized trial. Radiother Oncol. 2013 Aug;108(2):197-202.

48 Livi L, Meattini I, Marrazzo L, Simontacchi G, Pallotta S, Saieva C, et al. Accelerated partial breast irradiation using intensity-modulated radiotherapy versus whole breast irradiation: 5-year survival analysis of a phase 3 randomised controlled trial. Eur J Cancer. 2015 Mar;51(4):451-63.

49 Vaidya JS, Joseph DJ, Tobias JS, Bulsara M, Wenz F, Saunders C, et al. Targeted intraoperative radiotherapy versus whole breast radiotherapy for breast cancer (TARGIT-A trial): an international, prospective, randomised, non-inferiority phase 3 trial [Internet]. Lancet. 2010 Jul;376(9735):91-102. Available from: https://linkinghub.elsevier.com/retrieve/pii/S0140673610608379

50 Wazer DE, Hepel JT, Riker AI, Harness JK Chung C, Khan AJ, et al. In regard to Vaidya et al. Int J Radiat Oncol Biol Phys. 2015 Aug 1;92(5):952-3.

51 Meattini I, Boersma L, Livi L, Kirkove C, Gabryś D, Somaiah N, et al. In regard to Vaidya et al. Int J Radiat Oncol Biol Phys. 2015 Aug 1;92(5):960-1. 